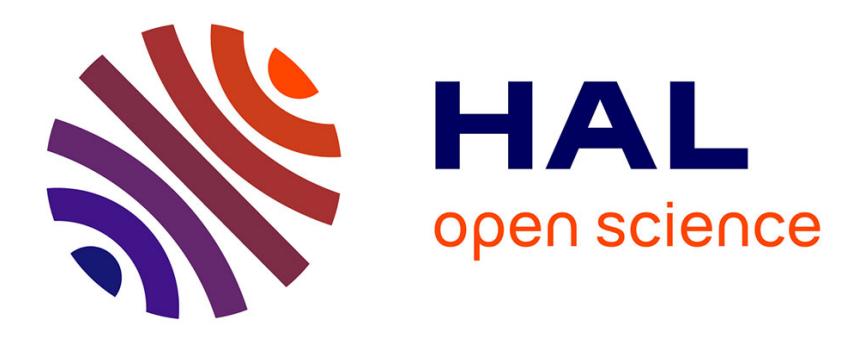

\title{
On the failure resistance of quasi-periodic lattices
}

\author{
Arthur Glacet, Julien Réthoré, Anne Tanguy, Fabrice Morestin
}

\section{To cite this version:}

Arthur Glacet, Julien Réthoré, Anne Tanguy, Fabrice Morestin. On the failure resistance of quasiperiodic lattices. Scripta Materialia, 2018, 10.1016/j.scriptamat.2018.07.001 • hal-03360645

\section{HAL Id: hal-03360645 \\ https://hal.science/hal-03360645}

Submitted on 30 Sep 2021

HAL is a multi-disciplinary open access archive for the deposit and dissemination of scientific research documents, whether they are published or not. The documents may come from teaching and research institutions in France or abroad, or from public or private research centers.
L'archive ouverte pluridisciplinaire HAL, est destinée au dépôt et à la diffusion de documents scientifiques de niveau recherche, publiés ou non, émanant des établissements d'enseignement et de recherche français ou étrangers, des laboratoires publics ou privés. 
On the failure resistance of quasi-periodic lattices

Arthur Glacet $^{\mathrm{a}}$, Julien Réthoré ${ }^{\mathrm{b}}$, Anne Tanguy ${ }^{\mathrm{a}}$, Fabrice Morestin ${ }^{\mathrm{a}}$

a LaMCoS, Université de Lyon / INSA Lyon / CNRS UMR 5259, Bat. Jacquard, 27 Avenue Jean Capelle, F-69621 Villeurbanne, Cedex, France

${ }^{\mathrm{b}}$ Civil and Mechanical Engineering Research Institute (GeM), Centrale Nantes / CNRS UMR 6183, 1 rue de la Noë, F-44321 Nantes, France

Abstract: Quasi-periodic materials have been widely studied for their behavior regard ing atomic dynamics, photonic, magnetic and electronic properties. They have unique properties inherited from their specific material symmetry. The recent development of additive manufacturing gives the opportunity to produce quasi-periodic structures to benefit from their unique capability. In this paper, quasi-periodic beam lattices are produced and failure experiments are performed. Then, a numerical model is proposed and validated. It is obtained that quasi-periodic Penrose lattices can outperform their periodic counterpart. These results open new ways to design architected materials with enhanced failure energy dissipation capabilities.

Keywords: lattice materials, quasi-periodic structure, failure, experiments, finite element

The recent development of additive manufacturing gives the opportunity to produce metamaterials like closed cellular materials efficiently,i.e. with a perfect control of the cell shape and distribution. As a particular case, lattice materials are interesting in many fields of application because of their low density. Due to this low density, the question regarding their mechanical properties and their integrity is important. While, their effective elastic properties and energy absorption capabilities under compression have been widely studied [1, 2], their failure behaviour remains almost unexplored except a very few papers as [3] concerning crack initiation.

The analysis of lattice materials is usually limited to periodic patterns. But, using additive manufacturing there is no limitation and quasi-periodic arrangements can be obtained. Considering that quasi-periodic structures have demonstrated unique properties regarding various physical phenomena (e.g. to store energy in local non-propagative vibration modes or to resist to the propagation of defects), it should be interesting to produce using additive manufacturing quasiperiodic lattices that inherit outstanding properties from their specific arrangement. Quasi-periodic materials have been widely studied for their behavior regarding atomic dynamic, photonic, magnetic and electronic properties $[4,5,6]$. They have unique properties inherited from their specific material symmetry. Indeed, quasi-crystals usually have high order large scale symmetry from which they inherit macroscopic isotropy for many physical properties. But contrary to periodic materials that hold the same order of symmetry whatever the observation scale, quasi-crystals appear almost amorphous at smaller scales.

In this paper, quasi-periodic beam lattices are produced at the macroscopic scale (typical beam length of $1 \mathrm{~mm}$ ) from a photo-sensitive ABS-type polymer powder and failure experiments are performed. Then, a numerical model using elastic Euler-Bernoulli beam elements and an energy based fracture criterion is proposed and validated against the experiments. This allows to investigate the failure resistance of some specific arrangements and the impact of characteristic geometrical features on the related energy dissipation. It is obtained that Penrose-type quasi-periodic lattices outperform their periodic counterpart. These results open new ways to design architected materials with enhanced failure energy dissipation capabilities.

The behavior of 2D lattice materials (honeycomb) is investigated. Three types of lattice are selected:

1. quasi-periodic Kite \& Dart Penrose tilling [7]

2. periodic approximate of the octogonal lattice [8]

3. periodic hexagonal lattice 
They are considered as lattices of elastic beams of rectangular section. Once the absolute size of a specimen is fixed, the remaining parameters to design such materials are: the unit cell size or beam length, the beam width and the constitutive material. If the constitutive material is linear and brittle then its properties are supposed to affect the overall behavior ( global displacement and force) of the lattice only to a scaling parameter. However, varying the ratio between the unit cell size and the specimen size would allow to evidence size effects [9]. Also, the beam width $e$ is an important parameter as it strongly affects the ratio between the flexural stiffness (varying as $e^{3}$ ) and tension stiffness (varying as $e$ ) of the beams. The stiffness ratio is thus expected to scale with $e^{2}$ which allows to span large investigation domains in terms of competition between bending and tension for limited variation of $e$. This is useful because the variation of $e$ are limited in practice due to the resolution of the manufacturing process (lower bound) and assumptions for beam kinematics (upper bound). The relative density of a lattice scales as $e$ and thus as $1 /(l / e), l / e$ being the slenderness of the beams. While the relative density is a meaningful parameter to compare different classes of materials, in the case of beam lattices, slenderness is helping to analyze the trends as it is the parameter governing the competition between flexural modes and tension modes. However, the relation between these two parameters is straightforward and the results can also be interpreted from the insight of the relative density. Note that for a given value of beam slenderness, the relative density for the octogonal lattice and the Kite \& Dart Penrose tilling are similar while the relative density for the hexagonal lattice is 0.6 times lower.

Experiments have been performed on samples obtained by additive manufacturing. They are made from photo-sensitive ABS-type polymer powder. The bulk material obtained from this process is isotropic. Its elastic behavior is defined by a Young's modulus of 1.4 GPa and a Poisson's ratio of 0.4. The sample design is the same as in [1] with a centered pre-crack oriented at $30^{\circ}$ with respect to the direction perpendicular to the loading axis (see Supplementary Material). The lattice structure is embedded by zones completely filled with the material. These zones are caught by the grips of the loading device to apply the remote displacement. The speed of the grips is $0.1 \mathrm{~mm} / \mathrm{min}$. This design allows for loading a central square part of $90 \mathrm{~mm}$ size with a classical tensile device under macroscopic uniaxial tension. Due to the crack angle of $60^{\circ}$ with respect to the loading axis, the crack tips are submitted to a mixed mode loading.

The samples are loaded until failure. Due to the high amount of elastic energy stored in the specimen, failure is unstable for the tested beam width of $0.2 \mathrm{~mm}$. As an illustration of the results, an image of a Kite \& Dart Penrose lattice after failure is presented in Fig. 1 (see Supplementary Material for other configurations). One clearly observes interactions between the crack and the structure of the material. It seems that specific features of the lattice (such as the one marked in blue in Fig. 1) induce a deviation of the crack. They could be named extra-tough features as this effect is obtained systematically. The deviation of the crack path induced by these specific features of the lattice makes the actual crack length longer than if the cracks were straight, resulting in a higher effective (from a macroscopic point of view) failure energy. Conversely, periodic structures have weak planes (lines) inducing directionality effects as illustrated in [9]. Even if zigzag patterns can be obtained in some cases resulting in an increase of the effective failure energy as well, in the tests we performed on periodic lattices, cracks follow straight paths meaning that no dissipation mechanisms induced by the architecture are activated.

For modeling these experiments on the materials described above, a simple numerical model is developed in a in-house MATLAB code. It consists of 2D beam elements under the assumption of Euler and Bernoulli. The constitutive material is assumed to be linear elastic and inertia effects are ignored. The joints between the elements are supposed to be perfect: infinitely rigid with no dissipation. The criterion for beam failure is based on the element-average strain energy density. It allows for weighting the contribution of tension force and bending moment with their actual energy contribution. The averaged strain energy density is denoted for element $i$ as

$\Phi_{i}=\frac{1}{2 m e s\left(\Omega_{i}\right)} \int \sigma: \epsilon d \Omega$

in the following. In this Equation, $\Omega i$ denotes the volume of element $i$ ( mes( $\Omega i$ ) being its size), : holds for double contraction of second order tensors and $\varepsilon$, respectively $\sigma$, is the small strain symmetric tensor, respectively Cauchy stress tensor. The maximum value that can be sustained by a beam before 
it fails is a material parameter, namely $\Phi_{c}$. Quasi-static simulations are performed and failure is accounted for using the following steps:

1. Elastic simulation of the lattice under a unit prescribed external load (load factor $\lambda=1$ )

2. detection of the beam imax having the highest averaged strain energy density Фmax

3. the load factor is adjusted so that Фmax equals $\Phi_{c}: \lambda^{2}=\Phi_{c} / \Phi m a x$

4. the amplitudes of the displacement and external loads computed in step 1 are scaled by $\lambda$

5. the results are saved and beam imax is removed from the lattice

6. go to step 1 while the lattice can handle external loading (Фmax $>0$ )

It is assumed that the behavior of the beams is purely brittle and the global response is adjusted through the load factor in terms of applied displacement and force. In the case when the mechanical response of the specimen is not stable under monotonic loading, snap back (decreasing displacement and force) can be obtained. This is the main difference between the numerical simulations and the experiments in which this instability results in a dynamical response of the specimen (because the displacement can only increases, the specimen "jumps", with no control on the applied loading, from a stable configuration to the one having the closest but higher prescribed displacement). However, as there is no initial kinetic energy in the system, it is expected that dynamical effects have a very limited influence on the results. The algorithm proposed above to drive the simulation is thus a reliable approximation of the actual loading conditions applied to the specimen. A Griffith like criterion was also tested but its prediction in terms of stability was not correct compared to the experiments. One can argue that such a criterion is based on the existence of a stress singularity at the crack tip. In the analysed materials herein, this is not the case as the ratio between the crack length and the beam length is low (around 10). Further, in [10], we have demonstrated that a gradient-elasticity model must be used to capture the macroscopic deformation of the lattice and this kind of continuum model is known to cancel out the singularity at the crack tip.

The model involves two material parameters: the Young's modulus $E$ and the critical averaged strain energy density $\Phi c$. The global response of the specimen scales as $E$ and thus, the only meaningful parameter is the ratio $\Phi c / E$. However, due to linear nature of the considered model, the crack path is supposed not to depend on this parameter. To simulate the experiments, the two components of the displacement are fixed for all the nodes within a narrow band (its width being the average beam length) along the bottom edge. For the nodes within a narrow band along the top edge, the horizontal displacement is fixed while the vertical displacement is assigned a unit value that is adjusted in step 4. The rotation degrees of freedom are left free. For validation purposes, the crack path obtained for a Kite \& Dart Penrose lattice is compared to the experimental result in Fig. 1 for $e=0.2 \mathrm{~mm}$. The corresponding average $/ / e$ ratio is 6.7 . The agreement between the simulation and the experiment is excellent, validating the failure criterion.

From the simulations, not only the crack path is obtained but also the macroscopic response (force (F) v.s displacement (U) ) that gives an insight in the effective behavior of the lattice. Using numerical simulations, it is also easy to perform a sensitivity analysis to the lattice parameters. The analysis is restricted herein to the beam width $e$. The influence of $e$ is analyzed in terms of crack path but also in terms of dissipated energy using the macroscopic force v.s. displacement response. The macroscopic response of specimens are normalized by the force and displacement at the onset of failure $\left(F_{0}\right.$ and $\left.U\left(F_{0}\right)\right)$. The results for the Kite \& Dart Penrose lattice are illustrated in Fig. 2. The response of the lattices is also compared to that obtained for a continuum material in the configuration tested above (a finite element crack propagation simulation is run in a linear isotropic elastic behavior assuming that the crack propagates at a constant mixed-mode stress intensity factor). It is clearly obtained that the response of the lattice deviates from that of a continuum showing the intrinsic ability of the quasi-periodic Penrose tilling to resist to defects. Further, it seems that decreasing $e$ the lattice has the ability to dissipate more and more energy. For a quantitative analysis, twice the area of the surface defined by the normalized force v.s. displacement curves is computed. Thanks to the considered normalization by $\mathrm{F}_{\mathrm{o}}$ and $\mathrm{U}\left(\mathrm{F}_{\mathrm{o}}\right)$, that corresponds to the ratio $R$ between the dissipated energy and the stored energy before failure occurs. If the macroscopic response is purely brittle then 
$R=1$. If $R<1$ then the initial energy in the system is higher than what the material is able to dissipate, the macroscopic response is unstable and the remaining energy returns to the loading device. If $R>1$ then the failure process needs more energy than what was initially available in the system. The macroscopic response is thus stable and more energy is required to achieved complete failure. Thanks to the normalization defined above, $R$ is an indicator of the ability of the lattice to dissipate energy during failure. The analysis is repeated for different (realistic) values of $e$ and for the three different lattices mentioned previously.

The results are summarized in Fig. 3. It is confirmed that decreasing $e$ increases the ability of the lattice to dissipate energy. Concerning the Kite \& Dart Penrose tilling, for $e=0.2 \mathrm{~mm}(l / e \approx 6.7), R$ is lower than 1 what confirms that the macroscopic response is unstable as in the corresponding experiment (shown in Fig. 1). For this lattice, a comparison of the crack path for two values of $e$ is given in Fig. 4. When $e$ is smaller the flexural stiffness of the beam is much lower than the tensile stiffness, thus allowing the beams to curve. This higher activation of the flexural deformation modes induces a rougher crack path, the crack being modified by locally tough patterns (highlighted in blue in Fig. 4): instead of following the orientation prescribed by the macroscopic loading (as it is the case for $e=0.5 \mathrm{~mm}$, the crack follows the orientation having the lower failure energy in the vicinity of the crack tip. Due to these deviations of the crack, the effective crack length is higher and the energy intrinsically dissipated by the lattice is higher. For a comprehensive analysis, small insets are included in Fig. 3 to show the repartition of the element average of the strain energy density for different lattices and slenderness. These results are obtained by loading the lattice under uniaxial tension without initial crack. For the hexagonal lattice, it is clear how increasing the slenderness leads to a transition between a tension dominated deformation mode to a bending dominated mode. For low slenderness, promoting tension modes, the beams aligned with the loading hold most of the strain energy density. Conversely, for higher slenderness, promoting flexural modes, most of the strain energy density is hold by beams not aligned with the loading. This transition is also evidenced for the octogonal lattice with well organized deforming structures whereas the Kite \& Dart Penrose lattice produces disordered arrangements of the strain energy density.

A remarkable dependence of $R$ with respect to the average slenderness $l / e$ of the lattice is obtained in Fig. 3. A linear trend is easily fitted on the curves plotted in Fig. 3 for the three materials. One should mention that $l / e$ being proportional to the inverse of the relative density of the lattice. Gibson-Ashby like trends are obtained for $R$, scaling with the inverse of the relative density (see Supplementary Material). It is obtained that the slope is higher for the Kite \& Dart lattice than for the two others: 1.5 times higher than for the octogonal lattice and 3 times higher than for the hexagonal lattice. One could argue that the structured deformation patterns (see Fig. 3) obtained for the hexagonal and octogonal lattices are one reason for the lower increase of the energy dissipation capability of these lattices. Conversely, deformation patterns for the Kite \& Dart Penrose lattice are disordered which is less favorable for the propagation of defects. Indeed, these structured strain energy density patterns obtained for a periodic lattice give rise to the existence of weak orientations [9] (crack propagation is promoted along some specific orientations defined by the unit cell geometry), or so called directionality effects that intrinsically weaken this kind of architecture. Not only the periodicity but also the order of local material symmetry, which is shown in [11] to be in close relation with dissipation mechanisms in disordered materials, might be at the origin of this difference between the Kite \& Dart Penrose that has a 5-fold symmetry and the hexagonal and octogonal lattices that have a 6-fold respectively 8-fold symmetry. As the octogonal lattice used herein is a periodic approximate of the quasi-periodic lattice, a it may also be affected by the existence of weak orientations locally. It is thus concluded that periodicity and high order symmetry affects the ability of a lattice to dissipate energy during the propagation of a crack. The higher performances of the Kite \& Dart lattice, are a consequence of disordered deformation patterns and of the activation of bending deformation modes when $e$ is decreasing that make some specific features of the lattice extra-tough.

The main result arising from this analysis is the evolution of ability of a given lattice to dissipate energy during failure varying beams' slenderness. A linear trend is obtained for the three lattices analyzed herein. While for a periodic lattice or a periodic approximate of quasi-periodic lattice, the slope of the linear trend is similar, it is 4 times higher for a quasi-periodic lattice based on the Kite \& Dart Penrose tilling. Analyzing the crack paths, it is observed that increasing the beam's slenderness allows bending deformation to develop. This results in deformation modes that prevent cracks from propagating through some specific patterns (such as the one marked in blue in Figs. 1,3) of the K\& D 
lattice making them extra-tough. This results in rougher crack paths and thus higher energy dissipation capabilities.

The supports of CNRS through METAMORPH PEPS project and ANR through grant ANR-16CE30-0007-01 are gratefully acknowledged. 


\section{References}

[1] W. Warren, A. Kraynik, Mechanics of Materials 6 (1) (1987) 27-37.

[2] L. J. Gibson, M. F. Ashby, Cambridge university press, 1999.

[3] I. Christodoulou, P.J. Tan, Engineering Fracture Mechanics 104 (2013) 140161

[4] J. Ashraff, R. Stinchcombe, Physical Review B 39 (4) (1989) 2670.

[5] Y. K. Vekilov, I. Gordeev, E. Isaev, Journal of Experimental and Theoretical Physics 89 (5) (1999) 995-999.

[6] A. Szallas, A. Jagannathan, Physical Review B 77 (10) (2008) 104427.

[7] R. Penrose, The Mathematical Intelligencer 2 (1) (1979) 32-37.

[8] M. Duneau, Journal of Physics A: Mathematical and General 22 (21) (1989) 4549.

[9] J. Réthoré, T. Dang, C. Kaltenbrunner, Journal of the Mechanics and Physics of Solids 99 (2017) 35-49.

[10] J. Réthoré, C. Kaltenbrunner, T. Dang, P. Chaudet, M. Kuhn, International Journal of Solids and Structures 72 (2015) 108-117.

[11] T. Damart, A. Tanguy, D. Rodney, Physical Review B 95, 054203 (2017). 
Figures: 

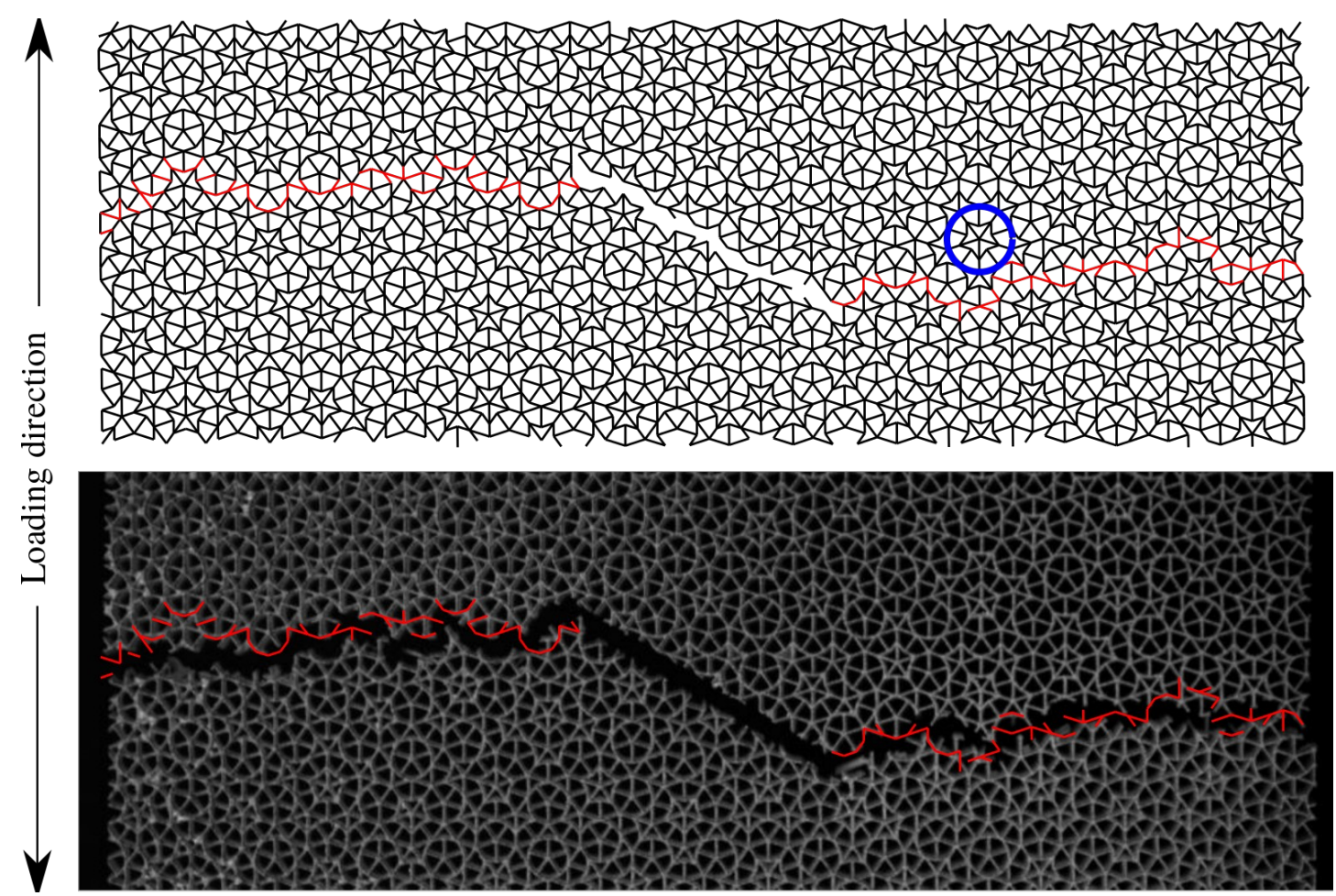

Figure 1: Comparison of the failure path between experiments and numerical simulations for the Penrose tilling for $e=0.2 \mathrm{~mm}$ (l/e $\approx 6.7)$. The loading direction is vertical and the crack orientation is $30^{\circ}$ with respect to the direction perpendicular to the load. The predicted failure path has been overlaid in red onto the experimental picture. The blue circle outlines one of the patterns in the lattice that were to induce a deviation of cracks. 


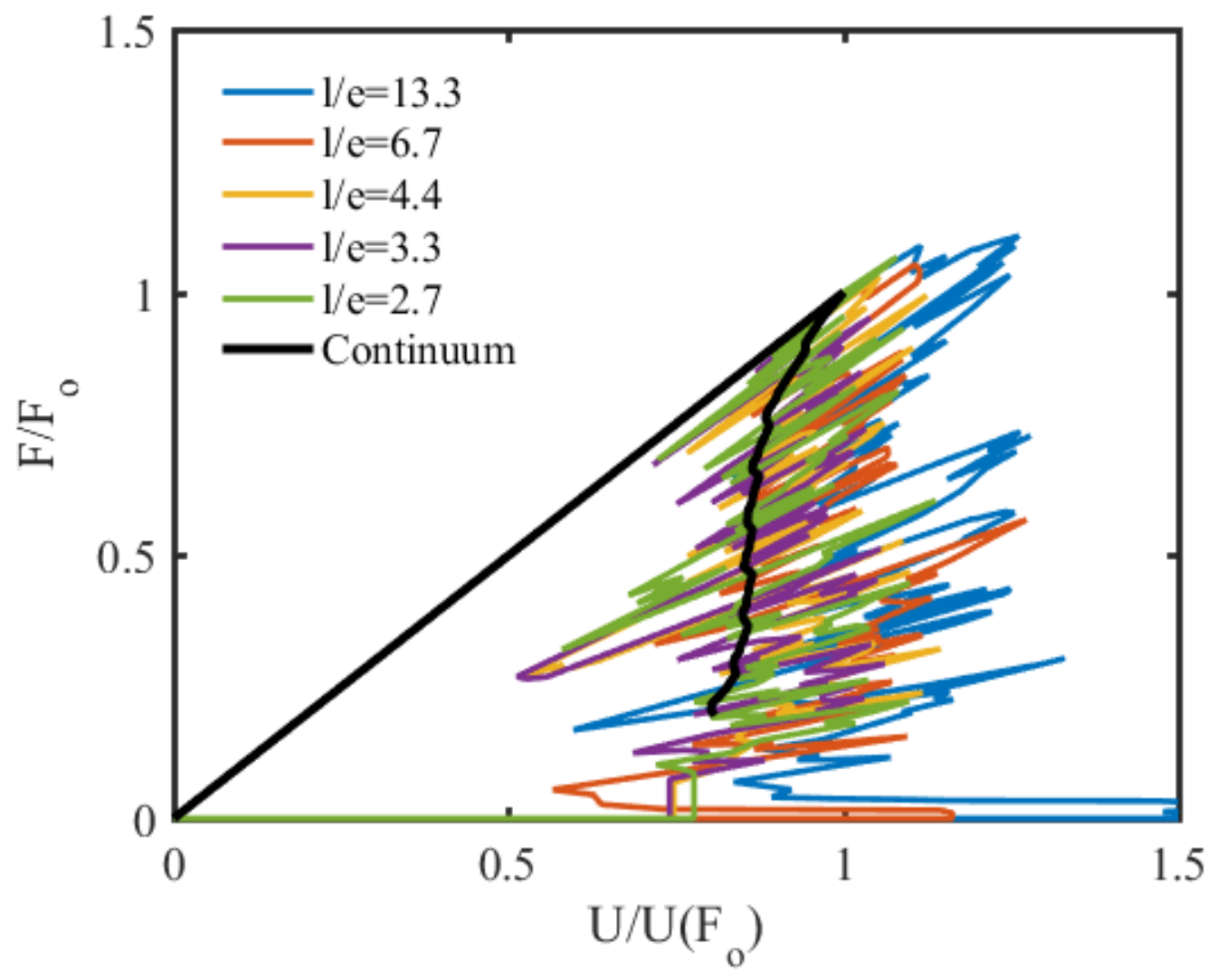

Figure 2: Scaled load $\left(\mathrm{F} / \mathrm{F}_{\mathrm{o}}\right)$ v.s. displacement $\left(\mathrm{U} / \mathrm{U}\left(\mathrm{F}_{\mathrm{o}}\right)\right)$ response of the Penrose tilling for different beam slenderness $\mathrm{l} / e$. 


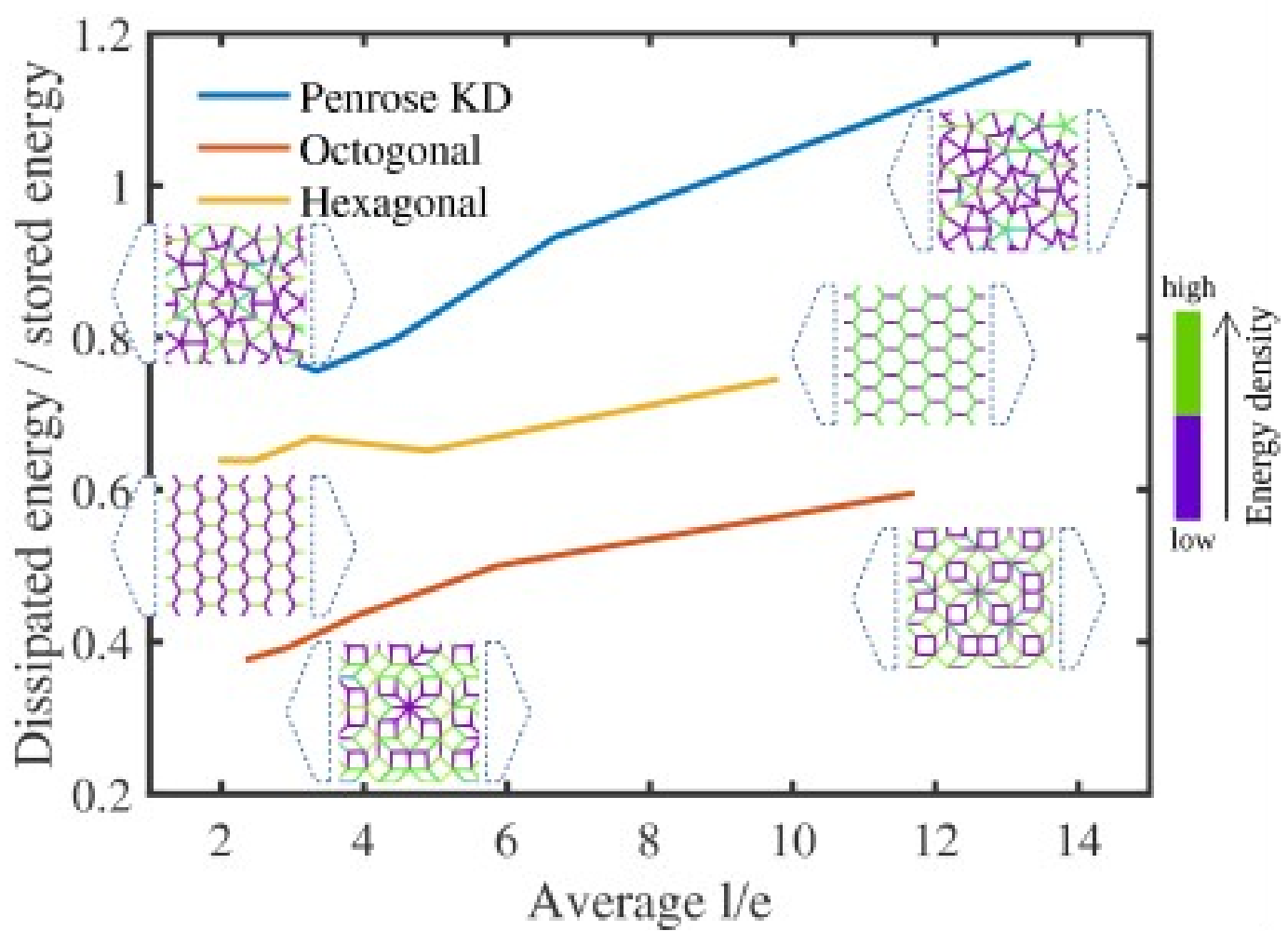

Figure 3: Evolution of the dissipated energy normalized with the elastic energy stored at failure initiation for different lattices with varying beam slenderness $l / e$. The insets show the repartition of the element average of the strain energy density for a uniaxial tensile test along the horizontal axis for different lattices and slenderness. Green beams hold high energy density whereas purple beams have low energy density. 

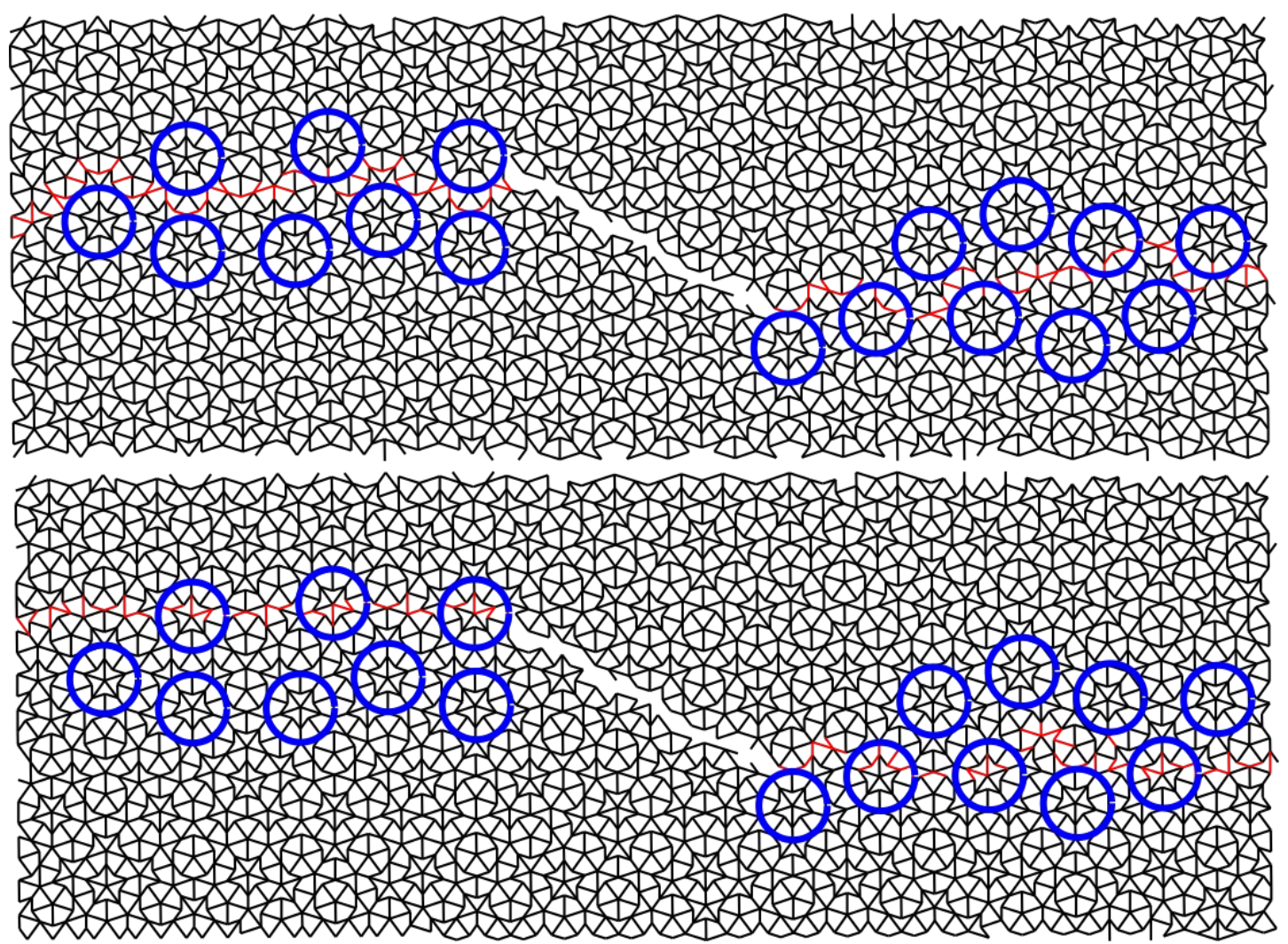

Figure 4: Comparison of the failure path for the Penrose tilling with different beam width: top $e=0.2 \mathrm{~mm}(1 / e \approx 6.7)$, bottom $e=0.5 \mathrm{~mm}(1 / e \approx 2.7)$. The patterns highlighted in blue are identified as those producing a deviation of the crack for higher slenderness. 